\title{
Performance comparison between dyes on single layered TiO2 dye sensitized solar cell
}

\begin{abstract}
Dye Sensitized Solar Cells (DSSC) is another kind of solar cell from the third generation that forms a photovoltaic. DSSC is designed to reduce cost from usage of expensive material in conventional solar panels. The purpose of this project is to fabricate and compare dye sensitized solar cells (DSSC) by using organic dye from blueberry and blue dye from chemical substances. The DSSC is fabricated using doctor Bladeômethod. Results are based on investigating the electrical performance and characteristic of the fabricated $\mathrm{TiO} 2$ solar cell based on these comparisons of dyes in order to investigate the potential of organic dyes as a light absorbing mechanism. The required data that is investigated are the open circuit voltage, Voc, short circuit current, Isc, fill factors, solar cells efficiency and UV absorption. Result shows good potential in the blueberry dyes as a sensitizer but further investigation is needed in order to fully understand the characteristic of these organic dyes.
\end{abstract}

Keyword: Absorbance; Blueberry; DSSC; Dye; Efficiency; Sensitizer; Solar cell 